\title{
Influenza viruses resistant to neuraminidase inhibitors
}

\author{
Aneta Nitsch-Osuch ${ }^{1 \otimes}$ and Lidia Bernadeta Brydak ${ }^{2}$ \\ 1Department of Family Medicine, Warsaw Medical University, Warsaw, Poland; 2Department of Influenza Research, National Influenza Center, \\ National Institute of Public Health - National Institute of Hygiene, Warsaw, Poland
}

\begin{abstract}
Neuraminidase inhibitors (NAls) are antiviral drugs for treatment and prophylaxis of influenza. By blocking the activity of the enzyme neuraminidase, NAls prevent new viral particles from being released. The increasing use of NAls brings into focus the risk of drug resistance arising to the class. There are three levels of antiviral resistance according to the way that resistance can be detected or inferred: genotypic, phenotypic and clinical resistance. For many years seasonal influenza viruses resistance to NAls was low (0.33\%). Recently, there has been described an increasing number of resistant seasonal influenza strains to oseltamivir (2\% in adults, $5-18 \%$ in children). In 2007 there were published data describing $14 \%$ resistant to oseltamivir strains of influenza $A / H 1 N 1 /$ in Europe. Approximately $0.5-1.0 \%$ of influenza $\mathrm{A} / \mathrm{H} 1 \mathrm{~N} 1 /$ pdm09 isolates are currently resistant to oseltamivir. The established markers of the resistance to oseltamivir were found in $\mathbf{2 . 4 \%}$ of human and $\mathbf{0 . 8 \%}$ of avian isolates of influenza $A / H 5 N 1 /$. It has been not observed a cross resistance among oseltamivir and zanamivir. NAls resistance in influenza viruses is relative and despite its presence patients with resistant viruses may still benefit from receiving these antivirals. The response to treatment with antivirals remains the most important proof of antiviral effectiveness. The rational use of NAls is essential to preserve the best choice for treatment and prophylaxis of seasonal, avian and pandemic influenza.
\end{abstract}

Key words: influenza, resistance, neuraminidase inhibitors Received: 18 May, 2014; revised: 03 September, 2014; accepted: 03 September, 2014 available on-line: 08 September, 2014

\section{INTRODUCTION}

Neuraminidase inhibitors (NAIs) are antivirals used both for the treatment and the prophylaxis of influenza. There are two well known neuraminidase inhibitors currently used: oseltamivir and zanamivir. The third NAI, peramivir, is available in the intravenous form and reserved for the critically ill patients. Another NAI, laninamivir, has received recently an approval in Japan (Thorlund et al., 2011). NAIs are effective against both types of influenza viruses (A and B), they are associated with less side effects and a better profile concerning drug resistance compared to the previously used another group of antivirals, the adamantanes (Reece, 2007). NAIs are anologes of the sialic acid, the natural substrate for viral neuraminidase (NA). By blocking the activity of the enzyme, NAIs prevent new viral particles from being released through the cleaving of terminal sialic acid on glycosylated haemagglutinin, and thus fail to facilitate virus releases (Moscona, 2005). The mechanism of action of
NAIs explains the necessity of the early usage of these drugs: 36-48 hours after the onset of clinical symptoms of the disease. The early initiation of treatment with NAIs provides the reduction of duration of symptoms and the lower frequency of secondary complications of influenza (Monto et al., 1999; Nicholson et al., 2000; Treanor et al., 2000). Several studies have also demonstrated that NAIs are effective in preventing seasonal influenza after exposure for close contacts, or as seasonal prophylaxis in the community (Hayden et al., 2000; Hayden et al., 2004). The recommended duration for antiviral treatment with NAIs is 5 days. Longer treatment courses for patients who remain severely ill after 5 days of treatment can be considered. The recommended duration of chemoprophylaxis with NAIs is 7 days (after last known exposure) (ACIP, 2014). Oseltamivir is also a drug of choice for treatment of influenza caused by highly and low pathogenic avian influenza viruses (HPAI and LPAI).

\section{RESISTANCE TO NAIS — GENERAL CONSIDERATIONS}

The problem of resistance of influenza viruses to NAIs is of the high importance and that's way there has been created the global Neuraminidase Inhibitor Susceptibility Network (NISN) which coordinates the analysis of clinical isolates collected thought the World Health Organization (WHO) surveillance network. Surveillance of the antiviral susceptibility of influenza viruses circulating in Europe has been established in 2004 though the European Union-funded European Surveillance Network for Vigilance against Viral Resistance (VIRGIL), in collaboration with European Influenza Surveillance Scheme (EISS), WHO and national influenza centers (Monto et al., 2006).

There are generally three levels of antiviral resistance according to the way that resistance can be detected or inferred (EMA, 2013):

- genotypic resistance (detecting though sequencing of the viral genome and identification of mutations previously associated with certain level of drug resistance);

- phenotypic resistance (resistance of the virus to drugs is tested in vitro by measuring viral replication at a different drug concentrations);

e-mail: anitsch@amwaw.edu.pl

Abbreviations: ACIP, Advisory Committee on Immunization Practices; CDC, Center for Diseases Control and Prevention; EISS, European Influenza Surveillance Scheme; EMA, European Medicine Agency; HA, haemagglutinin; HPAl, Highly Pathogenic Avian Influenza; LPAI, Low Pathogenic Avian Influenza; NA, neuraminidase; NAls, neuraminidase inhibitors; NISN, Neuraminidase Inhibitor Susceptibility Network; VIRGIL, Surveillance Network for Vigilance against Viral Resistance; WHO, World Health Organization 
- clinical resistance (based on animals and human patients and measuring or observing the actual response to treatment with antivirals).

Resistance to NAIs may be due to mutations in haemagglutinin (HA), which often confers resistance to both zanamivir and oseltamivir, while mutation in neuraminidase (NA) may render oseltamivir ineffective, but retains susceptibility to zanamivir (Gubavera, 2004). NA mutations selected from in vitro and in vivo experiments are limited to several conserved or semiconserved residues: R292K and E119G/A/D/V in N9 and N2 subtypes, H274Y in the N1 subtypes; and E119G, D198N, R152K in influenza B virus NA (Mc Kimm-Breschilin, 2000, Yen et al, 2006). Influenza virus variants with the N294S substitution in NA were also isolated after oseltamivir treatment from patients infected either by influenza A/ $\mathrm{H} 3 \mathrm{~N} 2$ / or influenza A/H5N1/ virus (Mc Kimm-Breschikin et al., 2000). In vitro studies have shown that mutations in both haemagglutinin and neuraminidase are associated with resistance development over prolonged passage (but the clinical significance of HA mutations is unknown) (Gubareva, 2004). The development of the resistance to oseltamivir is more common among seasonal influenza $\mathrm{A} / \mathrm{H} 1 \mathrm{~N} 1 /$ viruses $(27 \%)$ compared to seasonal influenza $\mathrm{A} / \mathrm{H} 3 \mathrm{~N} 2 /(3 \%)$ or $\mathrm{B}$ viruses (ACIP, 2014).

It should be underlined that oseltamivir resistance in influenza viruses is relative, and despite its presence patients with oseltamivir resistant viruses may still benefit from receiving oseltamivir. The clinical response to treatment with antivirals remains the most important proof of antiviral effectiveness (EMA, 2013). The clinical course of influenza in patients with resistant viruses is similar to the clinical course of the disease in patients carrying fully sensitive strains. However, it has been found that pneumonia occurs more often in patients from whom resistant influenza strains have been isolated (Okomo et al., 2013). Physicians must be aware that patients receiving antiviral medications, who do not respond to treatment, might have an infection with an resistant influenza virus. Oseltamivir resistance, sometimes within 1 week of treatment initiation, has been reported particularly among immunocompromised patients with influenza A/ $\mathrm{H} 1 \mathrm{N1} / \mathrm{pdm} 09$ virus infection, who were receiving the treatment with oseltamivir (CDC, 2014). Infection control measures are especially important for immunocompromised patients to reduce the risk for transmission of oseltamivir resistant viruses. Zanamivir is the treatment of choice for all patients where oseltamivir resistance is demonstrated or highly suspected. Intravenous zanamivir or peramivir may be considered where available (ACIP, 2014).

\section{SEASONAL INFLUENZA VIRUSES RESISTANCE TO NAIS}

Before 2009 influenza pandemic, the incidence of resistant viruses in patients treated with oseltamivir was estimated for $1-2 \%$ in adults and $5-6 \%$ in children (Whitley et al., 2001). Predominant mutations of NA in influenza $\mathrm{A} / \mathrm{H} 3 \mathrm{~N} 2 /$ virus were these resulted in an amino acid substitutions described as Arg292Lys and Glu119Val. Oseltamivir resistant virus was also isolated from $16.3 \%$ of Japanese children treated with oseltamivir for influenza in 2004 (Ward et al., 2005). All were due to mutations resulted in a substitution of histidine by tyrosine at the residue 274 (H274Y) in influenza A/ H1N1/ virus (Ward et al., 2005). The authors attributed the higher incidence of oseltamivir resistance to high viral titers and protracted viral shedding, more rigorous detection methods or relatively lower oseltamivir dosage ( $4 \mathrm{mg} / \mathrm{kg}$ regardless of the body mass). This high rate of resistance to oseltamivir should be treated as a serious warning of the possible overuse of NAIs in other countries. According to NISN data from years 19992002, among 2287 of influenza isolates only $0.33 \%$ had a reduced susceptibility to oseltamivir. None of these isolates were taken from patients who were known to have received NAIs. The significance of the resistant strains observed in individuals who were not exposed to oseltamivir is unclear at the present time. Such a situation was also described in 2007/2008 season in Europe. During this season, an oseltamivir resistant seasonal influenza A/H1N1/ strain with an H274Y substitution emerged in the northern hemisphere and spread rapidly around the world. In contrast to an earlier evidence of such resistant viruses being unfit, this resistant virus remained fully transmissible and pathogenic, and became the major seasonal A/H1N1/ virus globally within a year (Mc Kimm-Bereschikin et al., 2013). Approximately $14 \%$ of European strains of influenza A/H1N1/ were resistant to oseltamivir, but sustained sensitive to zanamivir (Lackenby et al., 2008). Oseltamivir resistance viruses have been detected in 9 countries (Denmark, Finland, France, Germany, Netherlands, Norway, Portugal, Sweden and United Kindom), in particular in Norway $(70 \%)$, France $(17 \%)$ Germany $(7 \%)$ and Great Britain $(5 \%)$, these resistant viruses carried the same mutation causing the substitution of histidine by tyrosine at residue $274(\mathrm{H} 274 \mathrm{Y})$ of the neuraminidase, which is known to confer a high level resistance to oseltamivir (Lackenby et al., 2008). The resistant variants (H274Y) have been isolated from both adults and children, ranging from 1 month to 61 years in age, with the majority of viruses being isolated from adults (Nicoll et al., 2008).

This resistant influenza A/H1N1/ virus with $\mathrm{H} 274 \mathrm{Y}$ substitution was displaced by the sensitive influenza A/ H1N1/pdm09 virus in the season 2009/2010. As of December 15, 2010, the WHO reported 314 samples of the prevalent influenza A/H1N1/pdm09 resistant to oseltamivir. Currently, approximately $0.5-1.0 \%$ of influenza A/H1N1/pdm09 isolates are resistant to oseltamivir (Mc Kimm- Bereschikin et al., 2013). During 2011 a new influenza $\mathrm{A} / \mathrm{H} 1 \mathrm{~N} 1 / \mathrm{pdm} 09$ variant with mildly reduced oseltamivir and zanamivir sensitivity was detected in more than $10 \%$ of community specimens in Singapore and more than $30 \%$ of samples from northern Australia (Hurt et al., 2011). Cases of resistance to oseltamivir have been observed mainly among immunosuppressed patients with prolonged viral replication during oseltamivir treatment, and persons who developed illness while receiving oseltamivir chemoprophylaxis (CDC, 2013, ACIP, 2014). Rare cases of infection with influenza A/ $\mathrm{H} 1 \mathrm{N1} / \mathrm{pdm} 09$ virus resistant, or with a reduced susceptibility to multiple neuraminidase inhibitors in severely immunosuppressed pediatric patients with a prolonged viral replication have also been reported (ACIP, 2014). The recently published meta-analysis of 15 studies revealed $2.6 \%$ of oseltamivir resistant influenza $\mathrm{A} / \mathrm{H} 1 \mathrm{~N} 1 /$ viruses and none strains resistant to zanamivir or peramivir (Thorlund et al., 2011). Some previously unreported amino acid substitutions have also been described: N325K substitution in influenza $\mathrm{A} / \mathrm{H} 1 \mathrm{~N} 1 / \mathrm{pdm} 09$ resulted in a reduced sensitivity to oseltamivir, G140R and N144K substitutions in influenza B caused a reduced sensitivity for oseltamivir, zanamivir and peramivir (Okomo et al., 2013).

The resistance of NAIs to zanamivir is much more rare than to oseltamivir. According to NISN report from 
1999-2002 only two isolates $(0.1 \%)$ had a reduced susceptibility to zanamivir. So far, zanamivir resistant viruses have not been isolated from immunocompetent individuals who have received zanamivir (Monto et al., 2006). One resistant virus was isolated from an immunocompromised child after bone marrow transplantation infected with type B influenza virus (Gubareva et al., 1998). It should be underlined that immunocompromised patients have difficulties with cleaning virus and this appears to promote a selection of drug resistance virus. As with oseltamivir, mutations that confer resistance to zanamivir may also reduce the virulence of the virus. Up to date, influenza virus strains which are in vitro resistant to oseltamivir stay susceptible to zanamivir. The lack of the cross resistance between oseltamivir and zanamivir may be explained by a longer use of oseltamivir and a limited number of zanamivir dosages (Reece, 2007). However, it is also possible that differences in the chemical structure of oseltamivir and zanamivir can result in different drug resistance profiles. Zanamivir more closely than oseltamivir resembles the natural substrate for NA the sialic acid), which may explain the lower resistance index (Varghese et al., 1998).

Although both drugs, zanamivir and oseltamivir, are based on the transition state analog of the sialic acid, zanamivir has a single substitution of a guanidine group at the 4'position on the sugar ring, whereas oseltamivir has an amino group at the 4 position, and also a bulky hydrophobic penthyl ether group replacing the glycerol side chains at 6'position (Varghese et al., 1997, Reece, 2007). It is also considered that differences in the mode of delivery and pharmacocinetics of NAIs have possible implications for the drug resistance. Lower drug concentrations at the site of viral replication (epithelial cells of the respiratory tract), observed after administration of oseltamivir compared to zanamivir, could enhance the risk by providing an environment for drug resistance virus to emerge (Reece, 2007).

\section{AVIAN INFLUENZA VIRUSES RESISTANCE TO NAIS}

According to the WHO classification there are highly pathogenic avian influenza viruses (HPAI), including influenza A/H5N1/, A/H7N7/, A/H7N9/, A/H10N8 and low pathogenic avian influenza viruses (LPAI), including influenza A/H9N2/ virus (WHO, 2014).

Oseltamivir is a drug of choice for the treatment of patients infected with influenza A/H5N1/ virus. Two different strains of highly pathogenic avian influenza A/H5N1/ have been circulating since 2003: clade 1 has been found in Vietnam, Thailand, Cambodia, Lao People's Democratic Republic and Malaysia; clade 2 emerged and spread from Peoples's Republic of China to Indonesia, Europe and Africa in 2004-2005 (WHO, 2007, Yuen \& Wong, 2005). It has been shown that compared to clade 1 isolates from 2004, some clade 1 Cambodian isolates and clade 2 Indonesian isolates from 2005 demonstrate a reduced sensitivity to oseltamivir (by phenotyping testing). The decrease in sensitivity may be due to drift mutations rather than from an exposure to oseltamivir (Yen et al., 2001; Tran et al., 2004; Chotpitayasununnondh et al., 2005). Some other reports describing the emergence of the resistance in influenza $\mathrm{A} / \mathrm{H} 5 \mathrm{~N} 1 /$ viruses isolated from patients in Vietnam and Egypt were published. Patients were treated with oseltamivir, and the resistant strains carried mutations causing substitutions H274Y or Asn294Ser (de Jong et al., 2005; Le et al., 2005; WHO, 2014). Up till now, the established markers of the resistance to oseltamivir were found in $2.4 \%$ of human and $0.8 \%$ of avian isolates of influenza A (H5N1) (Govorkova et al., 2013). Regarding other than influenza A (H5N1) viruses, some data indicate that an influenza A/H7N9/ isolate, encoding the R292K substitution, is highly resistant to oseltamivir and peramivir, and partially resistant to zanamivir (Sleeman et al., 2013).

\section{CONCLUSIONS}

The resistance to NAIs among influenza viruses is an emerging problem of the high epidemiological and clinical impact. The rational use of NAIs is essential to preserve the best choice for treatment and prophylaxis of seasonal, avian and pandemic influenza.

\section{REFERENCES}

Advisory Committee on Immunization Practices (ACIP) (2014) Prevention and Control of Seasonal Influenza with Vaccines. Recommendations of the Advisory Committee on Immunization Practices-United States, 2013-14. http://www.cdc.gov/flu/professionals/ acip/index.htm.(accessed 24 April 2014).

Chotpitayasununondh T, Ungchusak K, Hanshaoworakuln W (2005) Human disease from influenza A (H5N1), Thailand, 2004. Emerg Infect Dis 11: 1158-1159.

European Medicines Agency (EMA) (2013) Updated review of influenza antiviral medicinal products for potential use during pandemic by the Committee for Medical Products for Human Use (CHMP) of the European Medicine Agency. (accessed 12 April 2013).

Govorkova E, Baranovich T, Seiler P, Armstrong J, Burnham A, Guan Y, Peiris M, Webby R, Webster R, (2013) Antiviral resistance among highly pathogenic influenza A (H5N1) viruses isolated worldwide in 2002-2012 shows need for continued monitoring. Antiviral Research 98: $297-304$.

Gubareva LV (2004) Molecular mechanisms of influenza viruses resistance to neuraminidase inhibitors. Virus Res 103: 199-203.

Gubareva LV, Matrosovich MN, Brenner MK, Bethell RC (1998) Evidence for zanamivir resistance in an immunocompromised child infected with influenza B virus. I Infect Dis 178: 1257-1262.

Hayden FG, Gubereva LV, Monto AS (2000) Inhaled zanamivir for the prevention of influenza in families. N Engl J Med 343: 1282 1289.

Hayden FG, Belshe R, Villanueva C (2004) Management of influenza in households: a prospective, randomized comparison of oseltamivir treatment with or without post-exposure prophylaxis. J Infect Dis 189: 440-449.

Hurt AC, Lee RT, Leang SK, Cui L, Deng YM, Phuah SP, Caldwell N, Freeman K, Komadina N, Smith D, Speers D, Kelso A, Lin RT, Maurer-Stroh S, Barr IG (2011) Increased detection in Australia and Singapore of a novel influenza A(H1N1)2009 variant with reduced oseltamivir and zanamivir sensitivity due to a S247N neuraminidase mutation Eurosurveillance 16: pii19884.

de Jong MD, Tran TT, Troung HK, VoMH, Smith GJ (2005) Oseltamivir resistance during treatment of influenza A (H5N1) infection. N Engl J Med 353: 2667-2672.

Lackenby A, Hungnes O, Dudman SG, Meijer A, Paget WJ, Hay AJ, Zambon MC (2008) Emergence of resistance to oseltamivir among influenza A (H1N1) viruses in Europe. Eurosurveillance 13: 1-3.

Le Q, Kiso M, Someya K, Sakai T, Nguen T, Ngyuen K, Pham N (2005) Avian flu: isolation of drug resistant H5N1 virus. Nature 437: 1108.

Mc Kimm-Breschikin JL (2000) Resistance of influenza viruses to neuraminidase inhibitors. Antivir Res 46: 1-17.

Mc Kimm-Breschikin JL, Williams J, Barrett S, Jachno K, McDonald M, Mohr PG, Saito T, Tashiro M (2013) Reduced susceptibility to all neuraminidase inhibitors of influenza H1N1 viruses with haemagglutinin mutations and mutations in non-conserved residues of the neuraminidase. J Antimicrob Chemother 68: 2210-2221.

Monto AS, Fleming DM, Henry D (1999) Efficacy and safety of the neuraminidase inhibitor zanamivir in the treatment of influenza A and B virus infection. J Infect Dis 180: 254-261.

Monto AS, McKimm-Breschikin JL, Macken C, Hampson A, Hay A (2006) Detection of influenza viruses resistant to neuraminidase inhibitors in global surveillance during the first 3 years of their use. Antimicrob Agents Chemother 50: 2395-2402.

Moscona AM: Neuraminidase inhibitors for influenza (2005) New Eng/ $J$ Med 353: 1363-7134 
Nicholson KG, Aoki FY, Osterhaus AD (2000) Efficacy and safety of oseltamivir in treatment of acute influenza a randomized controlled oseltamivir in treatment of acu
trial. Lancet 355: 1845-1850.

Nicoll A, Ciancio B, Kramarz P (2008) Observed oseltamivir resistance in seasonal influenza viruses in Europe interpretation and potential implications. Eurosurveillance 13: 3-6.

Okomo-Adhiambo M, Sleeman K, Lysen C, Nguyen XT, Xu X, Klimov AI, Gubareva LV (2013) Neuraminidase inhibitor susceptibility surveillance of influenza viruses circulating worldwide during 2011 Southern Hemisphere season. Influenza Other Respir Viruses 7: 645658.

Reece PA (2007) Neuraminidase inhibitor resistance in influenza viruses. J Med Virol 79: 1577-1586.

Sleeman K, Guo Z, Barnes J, Shaw M, Stevens J, Gubavera LV (2013) R292K substitution and drug susceptibility of influenza A (H7N9) viruses. Emerg Infect Dis 19: 1521-1524.

Thorlund K, Award T, Boivin G, Thabane L (2011) Systematic review of inf.luenza resistance to the neuraminidase inhibitors. BMC Infect Dis 11: 134-142.

Tran TH, Nguen TL, Luong TS, Pham PM (2004) Avian influenza A (h5N1) in 10 patients in Vietnam. N Engl J Med 350: 1179-1188.

Treanor JJ, Hayden FG, Vrooman PS (2000) Efficacy and safety of the oral neuraminidase inhibitor oseltamivir in treating acute influenza: a randomized controlled trial. JAMA 283: 1016-1024.
Varghese JN, Smith PW, Sollins SL, Blick TJ (1998) Drug design against a shifting target: a structural basis for resistance to inhibitors in a variant of influenza virus neuraminidase. Structure 6: 735-746.

Ward P, Small I, Smith Jm Suter P, Dutkowski R (2005) Oseltamivir (Tamiflu) and its potential for use in the event of an avian influenza pandemic. J Antimicrob Chemother 55: i5-25.

Whitley Rj, Hayden FG, Reisinger KS, Young N, Dutkowski R (2001) Oral oseltamivir treatment of influenza in children. $J$ Pediatr Infect Dis 30: 127-133.

World Health Organization (WHO) (2014) Issues new guidelines for the avian flu virus.www.who.ont (accessed 24 April 2014).

World Health Organization (WHO) (2007) Avian influenza situation in Egypt - update, 18 January 2007.www.who.int/csr/don/2007.(accessed 24 April 2014).

Yen HL, Hoffmann E, Taylor G, Scholtissek C, Monto AS, Webster RG, Govorkova E (2006) Importance of neuraminidase active-site residues to the neuraminidase inhibitor resistance of influenza viruses. J Virol 80: 8787-8795.

Yen HL, Monto AS, Webster RG, Govorkova EA (2001) Virulence may determine the necessary duration and dosage of oseltamivir treatment for highly pathogenic A /Vietnam/1203/04 influenza virus in mice. Antimicrob Agents Chemother 192: 665-672.

Yuen KY, Wong SS (2005) Human infection by avian influenza A H5N1. Clin Microbiol Rev 11: 189-199. 